\title{
La evolución de los textos de pregrado de finanzas corporativas
}

Mauricio Avellaneda Hortúa*

* Magíster en Finanzas y mBA. Docente-Investigador, CIPE-ODEON, Universidad Externado de Colombia, Bogotá (Colombia). [mauricio.avellaneda@uexternado.edu.co].

Artículo recibido el 04 de noviembre de 2017.

Aceptado el 30 de noviembre de 2017.

Para citar este artículo:

Avellaneda Hortúa, M. (2017). La evolución de los textos de pregrado de finanzas corporativas. ODEON, 13, pp. 99-120.

DOI: https://doi.org/10.18601/17941113.n13.06 



\section{Introducción}

El 3 de marzo de 2017, a la edad de 73 años, falleció en Old Lyme, New London, Connecticut (Ee.uu.), el profesor Stephen Alan Ross. Como lo expresa la nota que con ocasión de su deceso publicó el Massachusetts Institute of Technology (мIT, 2017), Ross es reconocido por sus aportes y contribuciones al desarrollo de la teoría del arbitraje y a la teoría de la agencia. En forma adicional, se destaca su coautoría en el modelo de tasas de interés Cox-Ingersoll-Ross, y del modelo binomial para la valoración de opciones ${ }^{1}$. Recientemente, había desarrollado el teorema de la recuperación ( $R T$, Recovery Theorem), técnica con la que es posible determinar el contenido predictivo de los precios del mercado. Como docente, se desempeñó no solo como profesor en la Escuela de Administración y Dirección de Empresas Sloan del MIT, donde tenía a su cargo la cátedra Franco Modigliani de Economía Financiera, sino también, en la Universidad de Yale y en la Escuela de Negocios de Wharton de la Universidad de Pensilvania (мIт, 2017). Dentro de las diferentes distinciones que el profesor Ross recibió a lo largo de su carrera se destaca, en 2015, el Premio en Economía Financiera del Deutsche Bank (Center for Financial Studies, 2015). Habría que agregar, que fue autor y coautor no solo de varios artículos especializados en economía y finanzas, sino también, de varios textos académicos sobre finanzas corporativas (Fornero, 2007).

Teniendo en mente lo anterior, el presente artículo pretende rendir homenaje a la labor de difusión que el profesor Ross efectuó de los fundamentos de la teoría financiera a través del libro de texto que en inglés lleva como título Corporate Finance, obra traducida al español bajo el título Finanzas corporativas. En vista de que la primera edición en inglés de Corporate Finance data de 1988 y en español de 1995, es de particular interés verificar si en estas dos últimas décadas, las temáticas que aborda la versión en español de dicho texto han cambiado como consecuencia de los avances de la teoría financiera o de la praxis de la actividad

\footnotetext{
1 Rubinstein (2006) recuerda que en la historia de las finanzas existen algunos casos en los que se aplica la ley de la eponimia de Stigler. De acuerdo con ese principio, a algunas personas se les otorga el crédito académico por hechos que fueron anticipados por otros durante años, décadas e incluso centurias, o que fueron desarrollados por otros en forma independiente y casi simultánea, pero que en raras ocasiones son reconocidos o mencionados. Dentro de estos se encuentra el modelo en tiempo discreto de valoración binomial de opciones Cox-Ross-Rubinstein, cuya solución sugirió inicialmente Sharpe (1978). En dicha obra, el lector interesado puede consultar el capítulo 14 (Warrants, Options, and Convertible Securities), y allí el apartado Valuation of a Call Option on a Stock with Simple Price Changes (Sharpe, 1978, pp. 366-71).
} 
financiera. Con la intención de responder al interrogante planteado, esta investigación se propone revisar tanto los contenidos de algunos de los primeros textos de finanzas como de obras contemporáneas que tengan por objeto de estudio las finanzas corporativas, y a partir de esa revisión establecer, no solo la relevancia, sino también los cambios en las temáticas tratadas en dichos textos.

Para su desarrollo este documento se divide en seis secciones. La primera, corresponde a la presente introducción. La sección segunda pone de presente el dinamismo de las finanzas, lo que supone un reto para la enseñanza. Mientras que la tercera sección se ocupa de revisar el contenido y la evolución de las temáticas tratadas en los primeros textos de finanzas orientados hacia el estudio de las finanzas corporativas, la cuarta se concentra en las diferentes ediciones en español de los textos contemporáneos de finanzas corporativas, en particular de Principios de finanzas corporativas y Finanzas corporativas. En seguida, la sección quinta presenta algunos de los temas que en el campo de las finanzas corporativas se encuentran pendientes de resolver y, por ende, se constituyen en las líneas futuras de investigación. De las conclusiones se ocupa la sexta y última sección.

\section{Las finanzas: un dinámico campo de estudio}

A finales de la década de 1960, específicamente en 1968, el profesor James C. van Horne (1938-), de la Universidad de Stanford, publicó Financial Management and Policy. Luego de efectuar un breve recuento de la evolución de las finanzas a lo largo del siglo Xx, en la primera edición de esa obra en español (1973), expresó:

\footnotetext{
En general, el estudio de las finanzas se ha transformado, pues de un mero proceso descriptivo ha pasado a ser una disciplina que comprende un análisis riguroso y una teoría normativa; de una actividad limitada principalmente a la obtención de recursos, a otra que comprende el control del activo, la asignación y el cuidado del valor de mercado de cada entidad; y de una actividad que daba primordial importancia al análisis de las relaciones externas de la empresa, a otra que concentra su atención en la adopción de decisiones internas [...] La función del gerente financiero es muy distinta de lo que era quince años atrás y de lo que será sin duda, dentro de otros quince (van Horne, 1973, pp. 6-7).
}

En este punto importa recordar que para inicios de la década de 1970, las finanzas de Estados Unidos entraron en crisis (Marichal, 2010). De una parte, el elevado déficit comercial junto con el creciente costo de la guerra de Vietnam y, de otra, la decisión del presidente de este país, Richard Nixon (1969-1974) de solicitar a la Reserva Federal emitir dólares para paliar la situación, generó dudas respecto

pp. $99-120 \cdot$ n. $^{\circ} 13 / 2017$ 
de la capacidad de Estados Unidos para honrar la paridad del dólar con el oro, que se estableció a razón de una onza de oro por USD 35. La especulación contra el dólar no se hizo esperar, y la demanda por oro se incrementó, situación que llevó a la Reserva Federal a desprenderse en forma paulatina de sus tenencias de oro. En este estado, el presidente Nixon, en reunión con sus asesores en Camp David, el 15 de agosto de 1971 decidió poner término a la ventanilla de cambios de oro por dólares, lo que dio inicio a una época de tipos de cambios flexibles. Así las cosas, "los acuerdos de Breton Woods habían llegado a su fin y comenzaba una nueva época con un grado mucho mayor de volatilidad financiera a escala internacional" (Marichal, 2010, p. 178).

Como consecuencia de la decisión del presidente Nixon, el mundo pasó de un ambiente de estabilidad financiera a uno de volatilidad, situación que dejó su huella en el campo de las finanzas. Así por ejemplo, Haugen (1999), citado por Romero (2005), clasificó el desarrollo de las finanzas en tres épocas: las finanzas antiguas, las modernas y las nuevas. En las finanzas antiguas, que cubren el periodo comprendido entre finales del siglo XIX y mediados de la década de 1950, el objeto de estudio se relacionó no solo con el análisis de los estados financieros, sino también, con la naturaleza de las obligaciones financieras. Luego, en las finanzas modernas, que corresponde al periodo comprendido entre mediados de las décadas de 1950 y 1970, el campo de estudio se relacionó con la valoración basada en el comportamiento económico racional. Por último, las nuevas finanzas, que inician a partir de mediados de la década de 1970, se caracterizan por el estudio de los mercados ineficientes. En forma similar a Haugen (1999), Rubinstein (2006) dividió la historia de la economía financiera en tres etapas: el periodo antiguo (anterior a 1950), el periodo clásico, que abarca las décadas de 1950 a 1980 y, por último, el periodo moderno cuyos inicios se remontan a la década de 1980.

La permanente evolución de cualquier área del conocimiento y, en este caso, de las finanzas, supone un reto para la docencia. Para ilustrar lo anterior, en un aparte del prólogo de la primera edición en español del libro de texto que lleva como título Finanzas corporativas (Ross, Westerfield y Jaffe, 1995, p. vii) se lee:

La docencia y la práctica de las finanzas corporativas se han convertido en un desafío cada vez mayor. Los últimos quince años han presenciado cambios fundamentales en los mercados e instrumentos financieros. No pasa un solo día sin un anuncio en la prensa financiera en relación con asuntos tales como adquisiciones, obligaciones chatarra, reestructuración financiera y compras apalancadas. Tanto la teoría como la práctica de las finanzas corporativas han progresado a una velocidad extraordinaria y nuestra enseñanza debe avanzar al mismo paso. 
Con la finalidad de identificar los cambios en las diferentes temáticas que abordan los libros de texto de finanzas corporativas, la siguiente sección se ocupa de revisar los contenidos de los primeros textos dedicados a ese campo de estudio.

\section{Tras los primeros textos de finanzas corporativas}

Al estudiar la evolución de los libros de texto de finanzas empresariales, Norgaard (1981) identificó la existencia de tres etapas: la primera, o fase organizacional, cubre el periodo comprendido entre 1860 y 1949; la segunda, que designó como fase de transición, abarca el periodo comprendido entre 1950 y 1959, y la tercera y última, fase gerencial y de valuación, que inició en 1960.

La fase organizacional se caracterizó por constituirse en una etapa en la que, de una parte, los libros de finanzas se dirigieron en particular a abogados y contadores, y de otra, el objeto de estudio se centró en el estudio de las grandes corporaciones, y, en menor medida, en el análisis, por ejemplo, de los indicadores financieros. Como lo refiere Norgaard (1981), uno de los primeros libros de texto que se publicó en Estados Unidos que se orientó al estudio de la gestión de las empresas, fue Corporation Finance (Greene, 1897). De su prefacio se desprende que la obra tiene por objeto el estudio, tanto de las razones que llevan a las empresas estadounidenses a utilizar diferentes mecanismos de financiación como al análisis de sus resultados. La obra, con una extensión de 181 páginas, se divide en los siguientes ocho capítulos: I. Bonos y acciones, II. Formas de la empresa corporativa, III. Bonos ferroviarios, IV. Empresas subsidiarias y sus valores, V. Contabilidad de la corporación, vi. Examen de los informes ferroviarios, vII. Política pública hacia las utilidades corporativas, vIII. Reorganización corporativa y quiebra. De ellos, tres ocupan el 51,1\% de la obra: vIII. Reorganización corporativa y quiebra $(17,6)$; III. Bonos ferroviarios (17\%), y vi. Examen de los informes ferroviarios (16,5\%) (Greene, 1897).

En forma posterior, Edward Sherwood Meade, de la Escuela de Negocios Wharton, de la Universidad de Pensilvania, publicó Corporation Finance (Meade, 1910), libro que se diseñó, de acuerdo con su autor, para el estudiante universitario, constituyéndose de esta forma en el primer libro de texto universitario de finanzas corporativas (Norgaard, 1981). Como lo explica Meade (1910), la obra tiene por objeto explicar e ilustrar los métodos utilizados en la promoción, capitalización, gestión financiera, consolidación y reorganización de los negocios de las corporaciones. El texto se divide en 33 capítulos, distribuidos en 458 páginas.

pp. $99-120 \cdot$ n. ${ }^{\circ} 13 / 2017$ 
Probablemente, y como consecuencia de las crisis de Estados Unidos de 1896 y 1907, el capítulo xxxII, que trata de la reorganización empresarial -manejo de la quiebra-, es la sección a la que destina mayor espacio (34 páginas), equivalentes al 7,4\% de la obra.

En 1920, Dewing publicó The Financial Policy of Corporations. En el prefacio, el autor indica que la finalidad de la obra se relaciona con el estudio tanto de la estructura financiera como de los problemas financieros de las grandes corporaciones (Dewing, 1920). Para su desarrollo, la obra consta de cinco volúmenes: I. Valores corporativos, II. Promoción, III. La administración del Ingreso, IV. Expansión, y V. Quiebra y reorganización. De estos cinco, los más extensos son el cuarto y el quinto volumen. Mientras que el cuarto (Expansión) se ocupa, no solo de las diferentes formas en que un negocio puede expandirse, sino también de las fuentes de financiación para llevar a cabo dicho proceso, el quinto volumen (Quiebra y reorganización) tiene que ver con la forma como una compañía en dificultades financieras puede rehabilitarse. Como ejemplo de esta situación, se refirió a las diferentes crisis de los ferrocarriles, acaecidas desde mediados de la década de 1850 y hasta mediados de la década de 1910 (Dewing, 1920). Como respuesta a la petición de diversos profesores de finanzas corporativas, quienes sugirieron la necesidad de contar con casos típicos para la discusión en clase, Dewing publicó en 1922, Problems to Accompany 'The Financial Policy of Corporations' (1922). En el prefacio de esta obra, Dewing señaló que muchos de los problemas y casos allí incluidos los había utilizado en sus cursos de finanzas corporativas en la Escuela de Negocios de Harvard. Los 72 problemas o casos que contiene el libro fueron agrupados en las siguientes seis partes: I. Problemas relacionados con la forma y carácter de los valores, 21 problemas; II. La promoción de la Corporación, 20 problemas; III. La administración financiera de las corporaciones, 11 problemas; IV. La expansión de las corporaciones, 6 problemas; V. Reorganización de las corporaciones, 6 problemas, y, por último; vi. Inversiones, 8 problemas (Dewing, 1922).

Si bien la fase organizacional se caracterizó por ser un periodo en el que los textos tenían un carácter eminentemente descriptivo, Norgaard (1981) refirió los textos de McKinsey y Meech (1923), Jamison (1927) y Stevens (1934) como excepciones a esa regla.

En el prefacio del libro Controlling the Finances of a Business (McKinsey y Meech, 1923) los autores precisan que este tiene por finalidad el estudio de los problemas cotidianos de la administración financiera que tienen lugar en una empresa en marcha. A diferencia de los textos de la época, el de McKinsey y Meech centra su atención en el control presupuestal y en la utilización de métodos contables y 
estadísticos, indispensables para la administración científica de las finanzas. Antes de concluir el prefacio, resaltaron que con la llegada de la gestión científica a la planeación y al control financiero, se abría la posibilidad para aplicar en el área de finanzas de las empresas, metodologías que han resultado beneficiosas, por ejemplo, en el campo de la producción. En este punto resulta pertinente recordar que en muchas ocasiones el avance de las finanzas ha estado ligado al desarrollo de otras áreas del conocimiento. Para ilustrar lo anterior baste recordar que Black y Scholes (1973), al explicar la fórmula para la valoración de opciones, indicaron que la ecuación diferencial que hallaron correspondía a la ecuación de transferencia de calor de la física. Otros ejemplos de las conexiones entre la física y las finanzas pueden encontrarse en Weatherall (2013).

Continuando con el texto de McKinsey y Meech (1923), este consta de 628 páginas, divididas en 40 capítulos agrupados en las siguientes ocho partes: I. Antecedentes de la Administración Financiera, II. Determinación de los requerimientos de capital, III. Fuentes y mecanismos para la obtención de capital, IV. Comercialización de valores, v. Control de los desembolsos de capital, vi. Control del crédito, VII. Control de los ingresos, y vIII. Organización de la administración financiera. De las mencionadas partes, tan solo tres concentran el 63,7\% del texto (III. Fuentes y mecanismos para la obtención de capital, 24,8\%; II. Determinación de los requerimientos de requisitos de capital, 23,6\%, y V. Control de los desembolsos de capital, 15,3\%).

En cuanto a la obra de Stevens (1934 el libro se encuentra dividido en tres partes: I. Organización financiera, II. Obtención de capital, y III. Administración financiera. Mientras que a la primera parte le dedica en forma aproximada un tercio del libro, y a la segunda una sexta parte, la tercera y última concentran cerca de la mitad de la obra. Al reseñar el texto de Stevens (1934), Eutsler (1936) destacó que este cubre, entre otros, los problemas de la organización y gestión financiera de las pequeñas y medianas empresas, un cambio importante frente a la costumbre de considerar únicamente las grandes corporaciones. Con respecto a la tercera parte del libro (Administración financiera), Eutsler (1936) destacó la relevancia que el autor da al presupuesto como una herramienta para el desarrollo de la gestión financiera. En forma adicional, recalcó el uso de indicadores financieros como una herramienta para la evaluación de la gestión financiera. Norgaard (1981) elogió el hecho de que Stevens (1934) utilizó, aunque de forma elemental, el concepto del costo promedio ponderado de capital.

En respuesta a la falta de un texto que incluyera casos prácticos de finanzas, Baker y Malott (1936), de la Universidad de Harvard, Escuela de Graduados de pp. $99-120 \cdot$ n. ${ }^{\circ} 13 / 2017$ 
Administración de Empresas, Fundación George F. Baker (hoy Harvard Business School) publicaron Introduction to Corporate Finance. La obra consta de ocho partes: I. La Corporación, II. Instrumentos financieros y estructura de capital, III. Obtención de fondos para una corporación, IV. Administración del capital de trabajo, V. Gestión de la capitalización de una corporación, vi. Valoración y fusión, VII. Recapitalización y reorganización, y vIII. Instituciones financieras. Luego de introducir cada una de las mencionadas partes, los autores plantean una serie de casos (en total 59) con los que buscaron, en forma práctica, desarrollar los conceptos esbozados. Así, el texto incluye casos y problemas relacionados, no solo con la estructura corporativa, instrumentos financieros, cuestiones de política financiera y de gestión, sino también incorpora las relaciones existentes entre las empresas y la ley, y entre las empresas y las instituciones financieras. De las ocho partes en que se encuentra dividida la obra, las siguientes tres concentran el 51,7\% del texto: v. Gestión de la capitalización de una corporación (18,3\%), vi. Valoración y fusión $(16,9 \%)$, y IV. Administración del capital de trabajo (16,6\%). En forma adicional, vale la pena recalcar que el primero de los cinco apéndices que contiene la obra se centra en el análisis de los estados financieros.

En pocas palabras, tal y como lo refiere Norgaard (1981), los textos de finanzas de la etapa organizacional se distinguen por su carácter eminentemente descriptivo y episódico. Por episódico se refiere al hecho de ocuparse de los principales acontecimientos que ocurren a lo largo del ciclo de vida de una empresa, como por ejemplo: promoción, organización inicial, emisión de valores, fusión, reorganización y quiebra. En esa época, los textos se encontraban dirigidos especialmente a los abogados y contadores, quienes para ese momento se encargaban de llevar a cabo las actividades mencionadas.

Luego de la fase organizacional siguió la etapa de la transición, que tuvo su desarrollo a lo largo de la década de 1950. El fin de la Segunda Guerra Mundial (1939-1945) llevó a que el objeto de estudio se orientara hacia las finanzas internas de la empresa, en particular a la administración del capital de trabajo, y las finanzas de corto plazo. Norgaard (1981) refiere a Introduction to Business Finance (Howard y Upton, 1953) como el primer texto para esa época. La obra consta de 565 páginas, distribuidas en seis partes y 23 capítulos. Las temáticas del texto son las siguientes: I. Organización financiera, II. Herramientas administrativas de finanzas: análisis financiero, III. Herramientas administrativas de finanzas: presupuestos y planificación financiera, Iv. Financiación de corto plazo, v. Financiación de mediano y largo plazo, y vi. Problemas financieros no recurrentes. De esas seis 
partes, la tercera y la cuarta ocupan individualmente el 23,2\% de la obra, lo que pone de manifiesto el cambio en el énfasis de los libros de finanzas.

Para finales de la década de 1950, los libros de texto empiezan a ser influenciados por la publicación de artículos teóricos. Para el efecto, Norgaard (1981) señaló la publicación a partir de 1946 de la revista especializada en finanzas Journal of Finance. En forma adicional, mencionó los trabajos de Deal (1951), Solomon (1959) y Markowitz (1952). El libro The Management of Corporate Capital (Solomon, 1959) consta de cuatro partes integradas por un total de veintidós artículos. Las temáticas del libro son las siguientes: I. Medición del valor de la inversión, II. El costo de la deuda y de los fondos de capital, III. Soluciones generales a las decisiones óptimas de inversión, y IV. Aspectos especiales de la gestión del capital.

A partir de la década de 1960, el componente matemático empieza a ganar protagonismo en los textos de finanzas. Con el fin de mostrar lo anterior, Norgaard (1981) cita a Weston, autor de Financial Management, quien fue el primero en tratar la contribución de Modigliani-Miller (1958) a la discusión del costo de capital. En forma adicional, resaltó el libro Analysis for Financial Decisions (Beranek, 1963) por su contenido cuantitativo. Este texto se encuentra dividido en los siguientes catorce capítulos: I. Introducción. Elementos del análisis financiero, II. Metas, decisiones y modelos, III. Racionamiento de recursos: proyectos de un solo periodo, IV. Racionamiento de recursos: proyectos de dos productos, v. Racionamiento de recursos: múltiples periodos, vi. Proyectos de varios periodos: riesgo e incertidumbre, vII. Oferta de recursos, VIII. Financiamiento a largo plazo y maximización del valor de mercado de las acciones comunes, IX. Estructura de capital y riesgo, x. Cuentas por cobrar y política de crédito, XI. El saldo de caja, XII. Simulación, XIII. Programación lineal - el método Simplex, y XIV. Análisis de varios periodos y programación lineal. De los 14 capítulos en que se divide la obra de Beranek (1963), los siguientes tres concentran el 26,1\% del texto: XI. El saldo de caja $(10,0 \%)$, viII. Financiamiento a largo plazo y maximización del valor de mercado de las acciones comunes $(10,6 \%)$, y x. Cuentas por cobrar y política de crédito $(15,5 \%)$.

\section{Textos modernos de finanzas corporativas}

De acuerdo con Fornero (2007), la década de 1980 presenció el surgimiento de dos libros de texto focalizados en finanzas corporativas, obras cuyos títulos originales en inglés son los siguientes: Principles of Corporate Finance y Corporate Finance. A continuación una breve reseña de estos.

pp. $99-120 \cdot$ n. ${ }^{\circ} 13 / 2017$ 
La primera edición en inglés del libro Principles of Corporate Finance se remonta a 1981. Sus autores, los profesores Richard Brealey (1936-), de la Escuela de Negocios de Londres, y Stewart Myers (1940-), del Instituto Tecnológico de Massachusetts. A partir de la octava edición, se les unió el profesor Franklin Allen (1956-), de la escuela de Negocios de Wharton de la Universidad de Pensilvania. En 1995, Brealey y Myers, junto con Alan Marcus (1949- ), del Colegio de Boston, desarrollan un nuevo texto, Fundamental of Corporate Finance (Fornero, 2007).

En cuanto a la obra Corporate Finance, su primera edición en inglés data de 1988. Los autores son los profesores Stephen Ross (1940-2017) de la Universidad de Yale y Randolph Westerfield (1941-) de la Universidad del Sur de California. Para la segunda edición se les unió el profesor Jeffrey Jaffe (1947-) de la Escuela de Negocios Wharton de la Universidad de Pensilvania. En forma posterior, en 1991 y 1996, Ross, Westerfield y Bradford Jordan (1957-), este último de la Universidad de Kentucky, publicaron respectivamente Fundamentals of Corporate Finance y Essentials of Corporate Finance. Para 2007, Ross, Westerfield, Jaffe y Jordan se unen y publican Corporate Finance: Core Principles and Applications. Como se aprecia, de los autores antes mencionados, únicamente el profesor Ross ha fallecido.

\section{Principios de finanzas corporativas}

Con el fin de determinar las temáticas y la importancia de los diferentes tópicos considerados en la versión española de Principles of Corporate Finance (Principios de Finanzas Corporativas), esta investigación consultó las ediciones españolas correspondientes a los años 1988 ( $2^{\underline{a}}$ ed.), 1993 (4⿳亠丷a ed.), 2003 (7aㅡ ed.), 2010

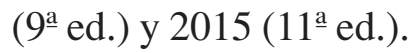

Al efectuar una breve reflexión respecto de la evolución del texto, a lo largo de las casi tres décadas que tiene la obra, los autores señalan lo siguiente:

La primera edición [en inglés] de este libro apareció en 1981. Los principios básicos son todavía los mismos, pero en las tres últimas décadas también se han generado cambios importantes en la teoría y en la práctica. La investigación financiera se ha concentrado menos en lo que los administradores financieros deberían hacer, y se ha enfocado en entender e interpretar lo que hacen en la práctica. En otras palabras, las finanzas se han vuelto más positivas y menos normativas (Brealey, Myers y Allen, 2015, p. viII). 
Al considerar los temas gruesos que se tratan en las referenciadas ediciones del texto en español Principios de Finanzas Corporativas (tabla 1), se aprecia que en todas ellas el texto se divide en once partes. Con respecto a los cambios en las temáticas incluidas se encuentra lo siguiente:

a. A partir de la edición de 1993, el estudio de las Opciones se constituye en una de las partes del texto, lo que pone de presente su relevancia. Es importante resaltar que en la edición de 1988, un fragmento de la temática de Opciones se encontraba inmerso en la parte VII Valoración de los diferentes tipos de deuda, específicamente en el capítulo 20, Los pasivos de la empresa y valoración de opciones.

b. Desde la versión de 2003, la Administración del riesgo se constituye en una de las partes del libro. En este punto vale la pena mencionar que la parte VIII Gestión del riesgo se integró con los capítulos 27, Gestión del riesgo y 28, Gestión de riesgos internacionales. Este último, con ligeras modificaciones, se hallaba en la edición de 1993, en la parte x Fusiones, finanzas internacionales y pensiones.

c. A partir de la publicación de 2003, las partes VIII Planificación financiera y IX Decisiones financieras a corto plazo, de las ediciones de 1988 y 1993, se consolidaron y dieron lugar, en la versión de 2003, a la parte Ix Planificación financiera y gestión a corto plazo.

d. Desde la edición de 2003 el texto incorpora temas relacionados con gobierno corporativo. Para ilustrar lo anterior, baste señalar que mientras en la versión de 1993, la parte x, Fusiones, finanzas internacionales y pensiones se encontraba dividida en tres capítulos, 33 Fusiones, 34 Gestión Financiera Internacional y 35 Planes de Pensiones, para la publicación de 2003, la parte x pasó a denominarse Fusiones, control y gobierno empresarial, parte que se dividió en los capítulos 33, Fusiones, y 34, Control, gobierno $\mathrm{y}$ arquitectura financiera.

En forma adicional hay que mencionar que los textos consultados tienen en promedio una extensión de 910 páginas, con desviación estándar igual a 155. En cuanto a su longitud se destacan las ediciones de 1993 y 2003 . La primera por su larga extensión (1123 páginas, que equivale al promedio adicionado de 1,37 veces la desviación estándar) y en el lado extremo, la segunda por su corta extensión (700 páginas, que corresponde al promedio disminuido en 1,35 veces la desviación estándar).

pp. $99-120 \cdot$ n. $^{\circ} 13 / 2017$ 
Tabla 1. Partes de Principios de finanzas corporativas

\begin{tabular}{|l|c|c|c|c|c|}
\hline \multicolumn{1}{|c|}{ Nombre de la parte y año de la edición } & $\mathbf{1 9 8 8}$ & $\mathbf{1 9 9 3}$ & $\mathbf{2 0 0 3}$ & $\mathbf{2 0 1 0}$ & $\mathbf{2 0 1 5}$ \\
\hline Introducción & 1 & & & & \\
\hline Valor & 2 & 1 & 1 & 1 & 1 \\
\hline Riesgo & 3 & 2 & 2 & 2 & 2 \\
\hline Las mejores prácticas en el presupuesto de capital & 4 & 3 & 3 & 3 & 3 \\
\hline Las decisiones de financiamiento y eficiencia de mercado & 5 & 4 & 4 & 4 & 4 \\
\hline Políticas de pago (de dividendos) y estructura de capital & 6 & 5 & 5 & 5 & 5 \\
\hline Opciones & & 6 & 6 & 6 & 6 \\
\hline Valoración de los diferentes tipos de deuda & 7 & & & & \\
\hline Financiamiento de la deuda & & 7 & 7 & 7 & 7 \\
\hline Administración del riesgo & & & 8 & 8 & 8 \\
\hline Planificación financiera & 8 & 8 & & & \\
\hline Planeación financiera y administración del capital de trabajo & 9 & 9 & 9 & 9 & 9 \\
\hline Fusiones, financiación internacional y pensiones & 10 & 10 & & & \\
\hline Fusiones, control corporativo y gobierno & & & 10 & 10 & 10 \\
\hline Conclusiones & 11 & 11 & 11 & 11 & 11 \\
\hline Número de páginas & 878 & 1123 & 700 & 976 & 871 \\
\hline
\end{tabular}

Fuente: elaborada por el autor a partir de Brealey y Myers (1988, 1993, 2003); Brealey, Myers y Allen (2010, 2015).

En lo que respecta a la importancia que la obra otorga a los diferentes temas tratados, la tabla 2 muestra para cada una de las versiones consideradas las tres partes que tuvieron una mayor dedicación, medida esta a partir del número de páginas que ocupa dentro de la obra. De la tabla 2 se destaca lo siguiente:

a. A lo largo de las ediciones consideradas, las partes de Valor y Política de pago de dividendos y estructura de capital son las que reciben una mayor atención. En la edición de 2015, la parte de Valor se divide en los seis capítulos que se relacionan a continuación: 1 . Introducción a las finanzas corporativas, 2. Cómo calcular valores presentes, 3. Valuación de bonos, 4. Valuación de acciones ordinarias, 5. Valor presente neto y otros criterios de inversión, y 6. Toma de decisiones de inversión con la regla del valor presente neto. Para esa misma edición, la parte de Políticas de pago de dividendos y estructura de capital se desarrolla en los siguientes cuatro capítulos: 1. Política de pago de dividendos, 2. ¿Es importante la política 
de endeudamiento?, 3. ¿Cuánto debe endeudarse una empresa?, y 4. Financiamiento y valuación.

b. En las versiones de 1988 y 1993, la parte vII, que en la primera se denominó Valoración de los diferentes tipos de deudas, y en la segunda Financiación por deuda, se constituyen en una de las tres principales temáticas de las mencionadas publicaciones, partes con similar contenido. Mientras que en la edición de 1988 esa parte se trató en cinco capítulos: 1. Los pasivos de la empresa y la valoración de opciones, 2. Valoración de la deuda con riesgo, 3. Los diferentes tipos de deuda, 4. Obligaciones convertibles y con certificado de opción, y 5. Arrendamiento (leasing), en la publicación de 1993 se redujo a los siguientes cuatro capítulos: 1. Valoración de la deuda con riesgo, 2. Los diferentes tipos de deuda, 3. Cobertura del riesgo financiero, y 4. Arrendamiento financiero (leasing).

c. Para las publicaciones de 2003 y 2010 la parte Ix, que en la primera llevó como título Planificación financiera y gestión a corto plazo, en la segunda se identificó como La planeación financiera y la administración del capital de trabajo, y se constituyó en una de las tres temáticas más tratadas en esas publicaciones. El contenido de ambas es similar. Mientras que en la versión de 2003, la parte vII se trató en los siguientes capítulos: 1. Valoración de la deuda, 2. Los diferentes tipos de deuda, y 3. Leasing (arrendamiento financiero), en la correspondiente a 2010 se abordó con los siguientes capítulos: 1. Análisis y planeación financieros, 2. Administración del capital de trabajo, y 3. Planeación financiera de corto plazo.

d. En la versión de 2010, la parte II, Riesgo, que ha estado presente en todas las ediciones de la obra, se constituye en una de las tres más importantes del texto, posición compartida en ese año con la parte de Planeación financiera y gestión de corto plazo. Su temática se desarrolla en los siguientes tres capítulos: 1. Introducción al riesgo, rendimiento y costo de oportunidad del capital, 2. Riesgo y rendimiento, y 3. Presupuesto de capital y riesgo.

e. Para la edición de 2015, la parte IV, Decisiones financieras y eficiencia del mercado, que ha estado presente en todas las ediciones de la obra, por primera vez se constituye en una de las tres más importantes de la obra. Esta parte se desarrolla en los siguientes tres capítulos: 1. Mercados eficientes y finanzas conductuales, 2. Panorama general del financiamiento corporativo, y 3 . Forma en la que las corporaciones emiten valores. 
Tabla 2. Relevancia de las partes tratadas (\%)

\begin{tabular}{|l|c|c|c|c|c|}
\hline \multicolumn{1}{|c|}{ Parte } & $\mathbf{1 9 8 8}$ & $\mathbf{1 9 9 3}$ & $\mathbf{2 0 0 3}$ & $\mathbf{2 0 1 0}$ & $\mathbf{2 0 1 5}$ \\
\hline Valor & 13,4 & 13,4 & 14,6 & 19,5 & 17,8 \\
\hline Política de pago (de dividendos) y estructura de capital & 12,8 & 12,8 & 12,9 & 14,0 & 12,9 \\
\hline Valoración de los diferentes tipos de deuda & 17,8 & 12,5 & & & \\
\hline Planeación financiera y gestión de corto plazo & & & 11,1 & 11,0 & \\
\hline Riesgo & & & & 11,0 & \\
\hline Decisiones financieras y eficiencia de mercado & & & & & 9,6 \\
\hline Suma & 44,0 & 38,7 & 38,6 & 55,5 & 40,3 \\
\hline
\end{tabular}

Fuente: elaborada por el autor a partir de Brealey y Myers (1988, 1993, 2003), y Brealey, Myers y Allen (2010, 2015).

\section{Finanzas corporativas}

Con el propósito de evaluar las temáticas y la importancia dada a los diferentes tópicos tratados en la versión española de Corporate Finance (Finanzas corporativas) esta investigación consultó las ediciones correspondientes a los años 1995 ( 3 ed.), 2000 (5 ed.), 2005 (7 ed.), 2009 (8 ed.) y 2012 (7 ed.). Conviene anotar que la edición de 1995 corresponde a la primera edición de la obra en español, traducción realizada a partir de la tercera edición en inglés del texto Corporate Finance. En la tabla 3 se presenta el detalle de las principales temáticas consideradas en las citadas ediciones. En ella se aprecia que mientras en las primeras ediciones el texto se dividió en siete partes, a partir de la publicación de 2005 consta de ocho partes.

En cuanto a la primera parte (Introducción, ediciones de 1995 y 2000, y Perspectiva General, ediciones de 2005, 2009 y 2012), se aprecian ligeras actualizaciones. Mientras que en las versiones de 1995 y 2000 la Introducción consta de dos capítulos: 1. Introducción a las finanzas corporativas, y 2. Estados financieros y flujo de caja o efectivo, en las publicaciones correspondientes a los años de 2005, 2009 y 2012 la parte de Perspectiva general se desarrolla en tres capítulos: 1. Introducción a las finanzas corporativas, 2. Estados financieros y flujos de efectivo, y 3. Planeación financiera y crecimiento. Con respecto al último capítulo, mientras que para la edición de 2009 se identifica como Análisis de estados financieros y planeación a largo plazo, para la del 2012 incorpora al análisis de estados financieros un par de subsecciones dedicadas a los modelos financieros y advertencias sobre los mismos, por lo que, en consecuencia, dicho capítulo adopta la denominación de Análisis de estados financieros y modelos financieros. 
A partir de la edición de 2005 ingresa a las grandes temáticas del texto, como parte vi, el tema de Opciones, futuros y finanzas corporativas, lo que indica la relevancia que este ha adquirido. Para la publicación de 2012, la parte de Opciones se desarrolla en cuatro capítulos: 1 . Opciones y finanzas corporativas, 2 . Opciones y finanzas corporativas: extensiones y aplicaciones, 3 . Warrants y bonos convertibles, y 4. Derivados financieros y cobertura de riesgo. En este punto es importante señalar que una parte del contenido de la sección vi (Opciones) de las ediciones de 2005 y siguientes, se originó en la parte v, Financiación a largo plazo, de las versiones de 1995 y 2000.

Tabla 3. Partes de Finanzas corporativas

\begin{tabular}{|l|c|c|c|c|c|}
\hline \multicolumn{1}{|c|}{ Nombre de la parte y año de la publicación } & $\mathbf{1 9 9 5}$ & $\mathbf{2 0 0 0}$ & $\mathbf{2 0 0 5}$ & $\mathbf{2 0 0 9}$ & $\mathbf{2 0 1 2}$ \\
\hline Introducción & 1 & 1 & & & \\
\hline Perspectiva general & & & 1 & 1 & 1 \\
\hline Valor y presupuesto de capital & 2 & 2 & 2 & 2 & 2 \\
\hline Riesgo & 3 & 3 & 3 & 3 & 3 \\
\hline Estructura de capital y política de dividendos & 4 & 4 & 4 & 4 & 4 \\
\hline Financiamiento a largo plazo & 5 & 5 & 5 & 5 & 5 \\
\hline Financiamiento a corto plazo & 6 & 6 & 7 & 7 & 7 \\
\hline Opciones, futuros y finanzas corporativas & & & 6 & 6 & 6 \\
\hline Temas especiales & 7 & 7 & 8 & 8 & 8 \\
\hline Número de páginas & 984 & 988 & 898 & 852 & 962 \\
\hline
\end{tabular}

Fuente: elaborada por el autor a partir de Ross, Westerfield y Jaffe (1995, 2000, 2005, 2009, 2012).

Con respecto a la extensión de Finanzas corporativas, el texto tiene, en promedio, 937 páginas, con desviación estándar de 60 páginas. Las ediciones con mayor y menor longitud corresponden respectivamente a las de los años 2000 y 2009. Mientras que la primera llega a 988 páginas (promedio adicionado de 0,85 desviaciones estándar), la segunda se sitúa en 852 páginas (promedio disminuido en 1,42 desviaciones estándar).

Acerca de la importancia relativa de los diferentes temas (partes) en que se divide el libro de Finanzas corporativas, la tabla 4 indica para cada una de las ediciones consideradas las tres partes que concentran el mayor número de páginas en la obra. De allí se observa lo siguiente: 
a. Las partes de Valor y presupuesto de capital, y Estructura de capital y política de dividendos, son las que en las cinco ediciones consultadas el texto da una mayor relevancia.

b. A partir de la edición de 2005, los temas de Riesgo, y Opciones, futuros y finanzas corporativas, ganan relevancia en la obra, temáticas que, como se señaló, estaban incorporadas en la parte de Financiación a largo plazo.

c. En este punto vale la pena resaltar que la importancia relativa de las tres temáticas más relevantes ha perdido participación (62,8\% edición de 1995 y 54,9\% edición de 2012), lo que sugiere que otras han ido ganando relevancia en forma paulatina.

Tabla 4. Relevancia de las partes tratadas (\%)

\begin{tabular}{|l|c|c|c|c|c|}
\hline \multicolumn{1}{|c|}{ Parte } & $\mathbf{1 9 9 5}$ & $\mathbf{2 0 0 0}$ & $\mathbf{2 0 0 5}$ & $\mathbf{2 0 0 9}$ & $\mathbf{2 0 1 2}$ \\
\hline Valor y presupuesto de capital & 20,3 & 19,6 & 19,6 & 18,4 & 22,1 \\
\hline Estructura de capital y política de dividendos & 22,2 & 22,3 & 22,7 & 20,4 & 19,5 \\
\hline Financiación a largo plazo & 20,3 & 21,9 & & & \\
\hline Riesgo & & & 12,9 & & 13,3 \\
\hline Opciones, futuros y finanzas corporativas & & & 12,9 & 13,0 & \\
\hline Suma & 62,8 & 63,8 & 68,1 & 51,8 & 54,9 \\
\hline
\end{tabular}

Fuente: elaborada por el autor a partir de Ross, Westerfield y Jaffe (1995, 2000, 2005, 2009, 2012).

\section{Tareas pendientes}

A pesar de los avances de la teoría financiera que se han visto incorporados en los textos de finanzas, en particular de finanzas corporativas, aún quedan varios temas por resolver. En el texto Principios de finanzas corporativas, los autores presentan en la última parte de la obra (Conclusiones) una relación de los conceptos o las ideas en finanzas que consideran conocer y desconocer. Por ser de particular interés aquellos problemas que se encuentran por resolver, sobre los que se dirige y orientará la investigación futura y los esfuerzos para lograr su solución, la tabla 5 presenta la relación de esos diez problemas. De los diez problemas identificados en la edición de 2015, el origen de ocho de ellos se remonta al siglo xx, y los dos restantes surgen en lo corrido del siglo xxi. Más aún, como consecuencia de la crisis financiera global que inició en 2007, en la última versión analizada (2015) los au- 
tores agregan a las problemáticas por resolver, la siguiente: “¿Por qué los sistemas financieros son tan propensos a las crisis?” (Brealey, Myers y Allen, 2015, p. 870).

Tabla 5. Lo que no sabemos: diez problemas no resueltos en finanzas

\begin{tabular}{|l|c|c|c|c|c|}
\hline \multicolumn{1}{|c|}{ Tema } & $\mathbf{1 9 8 8}$ & $\mathbf{1 9 9 3}$ & $\mathbf{2 0 0 3}$ & $\mathbf{2 0 1 0}$ & $\mathbf{2 0 1 5}$ \\
\hline ¿Cómo se adoptan las principales decisiones financieras? & 1 & 1 & & & \\
\hline $\begin{array}{l}\text { ¿Qué es lo que determina el riesgo de un proyecto y su valor } \\
\text { presente? }\end{array}$ & 2 & 2 & 1 & 1 & 1 \\
\hline El rendimiento y el riesgo: ¿qué nos ha faltado? & 3 & 3 & 2 & 2 & 2 \\
\hline $\begin{array}{l}\text { ¿Qué importancia revisten las excepciones a la teoría de los } \\
\text { mercados eficientes? }\end{array}$ & 4 & 4 & 3 & 3 & 3 \\
\hline La administración, ¿es un pasivo fuera del balance general? & & 5 & 4 & 4 & 4 \\
\hline $\begin{array}{l}\text { ¿Cómo podemos explicar el éxito de los valores y mercados } \\
\text { nuevos? }\end{array}$ & & 6 & 5 & 5 & 5 \\
\hline ¿Cómo se valoran las opciones complejas? & 5 & & & & \\
\hline $\begin{array}{l}\text { ¿Cómo podemos explicar los procedimientos de emisión de } \\
\text { acciones ordinarias? }\end{array}$ & 6 & & & & \\
\hline ¿Cómo podemos explicar la estructura de capital? & 7 & 7 & & & \\
\hline $\begin{array}{l}\text { ¿Cómo podemos solucionar la controversia del pago de } \\
\text { dividendos? }\end{array}$ & 8 & 8 & 6 & 6 & 6 \\
\hline ¿Qué riesgos debe asumir una empresa? & & & 7 & 7 & 7 \\
\hline ¿Cuál es el valor de la liquidez? & 9 & 9 & 8 & 8 & 8 \\
\hline ¿Cómo podemos explicar las olas de fusiones? & 10 & 10 & 9 & 9 & 9 \\
\hline $\begin{array}{l}\text { ¿Cómo podemos explicar las diferencias internacionales en la } \\
\text { arquitectura financiera? }\end{array}$ & & & 10 & 10 & \\
\hline ¿Por qué los sistemas financieros son tan propensos a las crisis? & & & & & 10 \\
\hline
\end{tabular}

Fuente: elaborada por el autor a partir de Brealey y Myers (1988, 1993, 2003), y Brealey, Myers y Allen (2010, 2015).

\section{Conclusiones}

Luego de una breve revisión de algunos textos de finanzas y, en especial, de finanzas corporativas, que cubre desde el trabajo de Greene (1897) hasta los textos de Ross, Westerfield y Jaffe (2012), y Brealey, Myers y Allen (2015), se aprecia el cambio del tipo de lector al que estas obras se dirigen. Así por ejemplo, mientras que el de Greene (1897) se orientó hacia abogados y contadores, los textos de Ross, Westerfield y Jaffe, y Brealey, Myers y Allen se dirigen primordialmente a estu- 
diantes de los cursos introductorios de finanzas corporativas, tanto en programas de pregrado como de posgrado, en este último caso a estudiantes de la maestría en Administración de Empresas. Teniendo en cuenta el carácter introductorio de los textos contemporáneos de finanzas corporativas, se requiere de los estudiantes conocimientos en áreas de contabilidad, estadística, economía y álgebra básica (Ross, Westerfield y Jaffe, 2012).

Al ser los textos Principios de finanzas corporativas y Finanzas corporativas introductorios al estudio de las finanzas corporativas, sus temáticas a lo largo del tiempo se han mantenido prácticamente inalteradas. Las principales novedades se relacionan, de una parte, con la incorporación y profundización en temas de administración del riesgo, opciones y futuros, fusiones, y control y gobierno corporativo. En forma adicional, al detallar el contenido de los citados textos se aprecia la inclusión de los últimos acontecimientos en los mercados financieros y, de esta forma, los textos enfatizan "los fundamentos modernos de la teoría de las finanzas y que esta cobre vida con ejemplos contemporáneos" (Ross, Westerfield y Jaffe, 2012, p. VII). Habría que decir también que ante un mundo más globalizado, los ejemplos ilustrativos han dejado de circunscribirse a Estados Unidos, de manera que su alcance sea universal.

Del enfoque empírico y descriptivo de los textos de finanzas empresariales de finales del siglo XIX hasta mediados del siglo Xx, los libros contemporáneos de finanzas corporativas se caracterizan por incorporar los fundamentos tanto de las finanzas modernas como de las nuevas finanzas (Romero Moreno, 2005), complementados y actualizados con situaciones prácticas circunscritas no solo a Estados Unidos.

Líneas futuras de investigación podrían orientarse a determinar tanto los avances como los asuntos pendientes que permitan, por ejemplo, resolver lo que Brealey, Myers y Allen (2015) han identificado como los temas de finanzas corporativas que a la fecha se encuentran pendientes de resolver.

\section{Referencias}

Baker, J. C. y Malott, D. W. (1936). Introduction to Corporate Finance. New York: McGraw Hill.

Beranek, W. (1963). Analysis for financial Decisions (Richard D. Irwin, ed.) Homewood, Illinois, USA. Recuperado de https://babel.hathitrust.org/cgi/pt?id=wu.8904844518 $3 ;$ view=1up;seq=17 
Black, F. y Scholes, M. (May-Jun de 1973). The pricing of options and corporate liabilities. The Journal of Political Economy, 81 (3), 637-54. Recuperado de http://www. eng.utah.edu/ cs6210/blackscholes.pdf

Brealey, R. A. y Myers, S. C. (1993). Principios de finanzas corporativas (4 ed.). Madrid: McGraw Hill.

Brealey, R. A. y Myers, S. C. (2003). Principios de finanzas corporativas (7 ed.). Madrid: McGraw Hill.

Brealey, R. A., Myers, S. C. y Allen, F. (2010). Principios de finanzas corporativas (9 ed.). México: McGraw Hill.

Brealey, R. A., Myers, S. C. y Allen, F. (2015). Principios de finanzas corporativas (11 ed.). México: McGraw Hill.

Brealey, R. y Myers, S. (1988). Principios de finanzas corporativas (2 ed.). Madrid: McGraw Hill.

Center for Financial Studies (2015). The Deutsche Bank Prize in Financial Economics. Frankfurt. Recuperado de https://www.ifk-cfs.de/fileadmin/images/10_db_prize/2015/db_prize_fyler_2015_LR.pdf

Deal, J. (1951). Capital Budgeting. New York: Columbia University Press.

Dewing, A. S. (1920). The Financial Policy of Corporations. Volume I. Corporate Securities. New York: The Ronald Press Company. Recuperado de https://archive.org/ details/cu31924024928099

Dewing, A. S. (1920). The Financial Policy of Corporations. Volume V. Failure and Reorganization. New York: The Ronald Press Company. Recuperado de https://archive. org/details/cu31924024928131

Dewing, A. S. (1922). Problems to Accompany 'The Financial Policy of Corporations'. New York: The Ronald Press Company. Recuperado de https://archive.org/details/ problemstoaccom00dewigoog

Eutsler, R. B. (January de 1936). Review Work of Financial Organization and Administration by W. MacKenzie Stevens. Southern economic Journal, 2 (3), 80-82. doi:10.2307/1053201 
Fornero, R. A. (2007). Cronología fotográfica de las finanzas. Los instrumentos, los conceptos, las herramientas. Universidad Nacional del Cuyo. Recuperado de http:// www.economicas.unsa.edu.ar/afinan/informacion_general/cronologia_finanzas/ cronologia_finanzas_volumen_5_1981_a_2007.pdf

Greene, T. L. (1897). Corporation Finance. New York: Putnam’s Sons.

Haugen, R. A. (1999). The Inefficient Sotck Market. What Pays Off and Why. New Jersey: Prentice Hall.

Howard, B. B. y Upton, M. (1953). Introduction to Business Finance. New York: McGrawHill. Recuperado de https://archive.org/details/introductiontobu00howa

Jamison, C. L. (1927). Finance. New York: The Ronald Press Company.

Marichal, C. (2010). Nueva historia de Las Grandes crisis Financieras. Una perspectiva global, 1873-2008. Bogotá: Sudamericana S.A.

Markowitz, H. (March de 1952). Portfolio Selection. The Journal of Finance, 7 (1), 77-91. Recuperado de https://www.math.ust.hk/ maykwok/courses/ma362/07F/ markowitz_JF.pdf

McKinsey, J. O. y Meech, S. P. (1923). Controlling the Finances of a Business. New York: The Ronald Press Company. Recuperado de https://babel.hathitrust.org/cgi/pt?id=u c1.\$b97912;view=1up;seq=7

Meade, E. S. (1910). Corporation Finance. Appleton and Company. Recuperado de https:// archive.org/details/corporationfinan00meadrich

MIT (6 de March de 2017). Professor Stephen Ross, inventor of arbirage pricing theory, dies at 73. мIT News. Cambridge, Massachusetts. Recuperado de http://news.mit. edu/2017/professor-stephen-ross-inventor-of-arbitrage-pricing-theory-dies-0306

Modigliani, F. y Miller, M. H. (June de 1958). The cost of capital, corporation finance and the theory of investment. The American Economic Review, 48 (3), 261-97. Recuperado de http://lib.cufe.edu.cn/upload_files/other/3_20140507105115_01.pdf

Norgaard, R. L. (1981). The evolution of business finance textbooks. Financial Management, 10 (2), 34-45. 
Romero Moreno, C. S. (2005). El pensamiento financiero: una visión de su desarrollo y sus fronteras. ODEON (2), 22-48.

Ross, S. A., Westerfield, R. W. y Jaffe, J. (2000). Finanzas corporativas. México: McGraw Hill.

Ross, S. A., Westerfield, R. W. y Jaffe, J. (2005). Finanzas corporativas (7 ed.). México: McGraw Hill.

Ross, S. A., Westerfield, R. W. y Jaffe, J. E. (2012). Finanzas corporativas (9 ed.). México: McGraw Hill.

Ross, S. A., Westerfield, R. W. y Jaffe, J. F. (1995). Finanzas corporativas (3 ed.). Madrid: Irwin.

Ross, S. A., Westerfield, R. W. y Jaffe, J. F. (2009). Finanzas corporativas (8 ed.). México: McGraw Hill.

Rubinstein, M. (2006). A History of the Theory of Investments. My annotated Bibliography. Hoboken: John Wiley y Sons.

Sharpe, W. F. (1978). Investments. New Jersey: Prentice-Hall.

Solomon, E. (1959). The Management of Corporate Capital. New York: The University of Chicago.

Stevens, W. M. (1934). Financial Organization and Administration. New York: American Book Company.

van Horne, J. C. (1973). Administración financiera. Buenos Aires: Contabilidad Moderna.

Weatherall, J. O. (2013). The Physics of Wall Street. A Brief History of Predicting the Unpredictable. New York: Houghton Mifflin. 